\title{
Sandra Regina Goulart Almeida
}

Universidade Federal de Minas Gerais

\section{Intervenções feministas: pós-colonialismo, poder e subalternidade}

\begin{abstract}
Resumo: À luz das teorias pós-coloniais e feministas, o artigo propõe uma reflexão sobre esses dois campos teóricos a partir tanto de suas articulações quanto de suas especificidades, procurando pensar ainda sobre suas relações com o contexto das teorias críticas produzidas no Brasil.
\end{abstract}

Palavras-chave: pós-colonialismo; feminismo; subalternidade.

Copyright (c) 2013 by Revista Estudos Feministas.

1 "No contexto pós-colonial global atual, nosso modelo deve ser o de uma crítica da cultura política, do culturalismo político, cujo veículo é a escrita de histórias legíveis, quer sejam hegemônicas ou alternativas" (Gayatri Chakravorty SPIVAK, 1989, p. 271, tradução nossa).

2 Rita Terezinha SCHMIDT, 2006, p. 756.
"In the current global postcolonial context, our model must be a critique of political culture, political culturalism, whose vehicle is the writing of readable histories, mainstream or alternative." Gayatri SPIVAK, 1989.

"O refinamento de habilidades interpretativas e o exercício da imaginação criativa são pré-condições para se construir a voltagem crítica e a autoridade intelectual da crítica feminista brasileira." ${ }^{2}$ Rita SCHMIDT, 2006.

As epígrafes que abrem esta reflexão, cada uma a seu modo, remetem ao ponto crucial de teorizações que se propõem a pensar o pós-colonialismo e o feminismo, suas imbricações e contendas, e sua relevante inserção no debate crítico na cena contemporânea, assim como o faz Deepika Bahri em seu conhecido e bem elaborado texto, "Feminismo in/and Postcolonialism", que a Revista Estudos Feministas traz em tradução para o português neste número. Levando em conta os argumentos trazidos pela autora indiana, radicada nos Estados Unidos, procuro, ao cotejar esses dois campos teóricos, refletir sobre as propostas e os desafios por eles trazidos, conjeturando, em especial, suas relações com o contexto das teorias críticas produzidas no Brasil. 
${ }^{3}$ SPIVAK, 1989, p. 276.

${ }^{4}$ SCHMIDT, 2006.

${ }^{5}$ Miriam ADELMAN, 2007, p. 394.

${ }^{6}$ ADELMAN, 2007, p. 395.
Uma reflexão sobre o pós-colonialismo e sua confluência com o feminismo necessariamente remete ao influente trabalho da crítica indiana Gayatri Chakravorty Spivak. Posicionando-se como uma teórica feminista e pós-colonial (destacando esses como sendo seus espaços enunciativos de afiliação e afetividade), Spivak elabora a proposta de um modelo de crítica pós-colonial que contempla fundamentalmente uma reflexão sobre os instrumentos de poder e as possibilidades de agenciamento do sujeito subalterno, sujeito esse marcadamente gendrado e que contribui para a "enorme complexidade do espaço pós-colonial, especialmente o espaço da mulher". ${ }^{3}$ Tal postura inquiridora a ser atribuída à forma de pensamento crítico aliada ao póscolonialismo, na visão da teórica, deveria se pautar pela maneira alternativa de pensar e ler os construtos históricos e culturais, assim como pelo questionamento dos protocolos de leitura e escrita e das construções discursivas que moldaram (e continuam moldando) obstinadamente o pensamento ocidental, consolidando-se ao longo do tempo.

Argumento similar aparece emoldurado nas palavras da crítica brasileira Rita Schmidt ao abordar a difícil inserção da crítica feminista na cultura letrada brasileira. Ao concluir seu artigo, Schmidt, fazendo coro às palavras de Spivak, evoca a necessidade premente de se valer, de maneira contundente e persistente, de habilidades interpretativas e críticas para que se possa consolidar um pensamento estratégico e intervencionista na crítica feminista brasileira. ${ }^{4}$

Depreende-se, assim, dessas duas reflexões judiciosas que as agendas pós-colonial e feminista comungam de princípios e crenças que trazem a possibilidade latente de um profícuo diálogo entre esses campos de análise crítica. Pode-se argumentar que a experiência feminista e a póscolonial, como observa Miriam Adelman, compartilham uma "epistemologia da alteridade", promovendo, assim, o resgate ou a releitura de "experiências invisibilizadas, silenciadas ou construídas como um Outro da modernidade ocidental". ${ }^{5}$ De fato, a ligação entre essas duas teorias críticas pode nos fornecer uma visão mais inclusiva de ambas as tendências e favorecer a produção de análises culturais, sociais e literárias que possam se articular entre si e que poderiam se configurar, como bem coloca Adelman, como sendo ao mesmo tempo feministas e pós-coloniais. ${ }^{6}$

Como sabemos, da mesma forma como ocorreu com relação a outras teorias ou movimentos críticos, o feminismo não se inseriu fácil e tranquilamente nos estudos pós-coloniais. Ao pensarmos, por exemplo, na literatura e no feminismo, vale ressaltar que são as críticas literárias feministas que abalaram, como registra Silviano Santiago, ao avaliar a posição do intelectual na contemporaneidade, os consolidados "pilares 
${ }^{7}$ Silviano SANTIAGO, 2004, p. 86.

${ }^{8}$ Heloisa Buarque de HOLLANDA, 1994, p. 8-9.

${ }^{9}$ Nelly RICHARD, 2002, p. 168.

${ }^{10}$ Stuart HALL, 1996, p. 264.

1 SCHMIDT, 2010, p. 270, grifos nossos. universais e seculares" da crítica e da literatura contemporânea, desarticulando, assim, "o sólido edifício das belles lettres". ${ }^{7}$ Nesse sentido, Heloisa Buarque de Hollanda salienta a importância que o pensamento feminista adquiriu, nessas últimas décadas, como expressão de uma "tendência teórica inovadora e de forte potencial crítico e político", mediante o que ela percebe como "a ineficácia dos discursos contestatórios da atualidade". ${ }^{8}$ Tal importância se deve ainda à interferência dos estudos da mulher e de gênero no rumo originalmente determinado para o desenvolvimento das teorias críticas contemporâneas, efetuando "um corte epistemológico nos blocos de saber/poder das disciplinas acadêmicas tradicionais", como nos lembra a crítica chilena Nelly Richard. ${ }^{9}$

O mesmo pode ser dito sobre a inserção do feminismo nos estudos culturais. Nas palavras de Stuart Hall, que evoca a emblemática metáfora da invasão e da interrupção como trabalho crítico para descrever o que ele avalia como sendo uma produtiva e fértil entrada enviesada do feminismo nos estudos culturais, "como um ladrão na noite, o feminismo forçou a entrada, causou uma interrupção, fez um estardalhaço, apoderou-se do momento", invadindo a seara dos estudos culturais. ${ }^{10} \mathrm{~A}$ concepção trazida pelo termo "interrupção", de fato, define de forma apropriada o fazer teórico da crítica feminista e se encontra também respaldada por Rita Schmidt em texto mais recente. Para a autora,

é indispensável acreditar que as energias feministas no
campo dos estudos literários têm a potencialidade de
interferir no discurso crítico, revitalizar o ensino e fecundar
uma agenda educativo-pedagógica-política capaz
de interromper as continuidades históricas das exclu-
sões, da violência e do preconceito. Essa interrupção
implica a desestabilização das estruturas patriarcais, a
transgressão de paradigmas binários, vigentes no
campo social e no campo científico, a descolonização
do pensamento em sentido amplo e irrestrito e a
reinvenção de subjetividades."

É justamente essa possibilidade de pensar o trabalho teórico como uma forma de interrupção teórica e epistemológica, como aquela efetuada não apenas pelos estudos feministas e de gênero, mas também pelos estudos sobre etnicidade e raça (campo especialmente articulado na crítica pós-colonial), que pode levar a uma desestabilização produtiva nas teorizações e nas análises da sociedade e das práticas culturais a ela associadas.

Nesse contexto, destaca-se o posicionamento da crítica feminista no sentido de tecer uma narrativa crítica dos construtos sociais e culturais, apossando-se do fazer crítico como forma de desestabilizar o poder instituído e de refletir sobre questões de poder, vitimização e agenciamento. 
${ }^{12}$ Mary EAGLETON, 2005, p. 5.

13 Susan Stanford FRIEDMAN, 1998.

${ }^{14}$ Para uma discussão sobre os conceitos de interseccionalidade e a transversalidade de gênero, ver Lia Zanotta MACHADO, 1998. Ambos os termos têm sido amplamente usados pela crítica feminista. A transversalidade de gênero pode ser vista como "o entendimento de que a construção social do gênero perpassa as mais diferentes áreas do social" (MACHADO, 1998, p. 108), não apenas em uma perspectiva que enfatiza a interrelação e a intersecção entre essas áreas, como no caso da interseccionalidade, mas principalmente pela percepção que tal inter-relação se dá de maneira transversal e mutuamente imbricada.

${ }^{15}$ RICHARD, 2002, p. 169.

${ }^{16}$ Ania LOOMBA, 1998, p. 229.
Ressalte-se que são justamente estas últimas questões que têm movido grande parte da crítica feminista: por um lado, procura-se elaborar um discurso sobre a vitimização histórica e o legado desse processo de silenciamento e invisibilidade; por outro, busca-se construir um conceito propositivo de agenciamento que possa incluir as condições existenciais e materiais das mulheres como uma categoria complexa, multifária, contingenciada pelos múltiplos constituintes identitários e pela existência inequívoca de "hierarquias de gênero, classe e raça", nas palavras da crítica feminista inglesa Mary Eagleton. ${ }^{12}$

Assim, discutir a crítica feminista na contemporaneidade requer que estejamos atentas às várias teorizações recorrentes nos últimos anos que fortaleceram a crença em um espaço plural de articulações de diferenças em suas múltiplas manifestações. ${ }^{13}$ Marcadamente vinculado com os vários feminismos, esse posicionamento destaca a necessidade de negociação entre os múltiplos constituintes identitários, a interseccionalidade e a transversalidade de gênero, ${ }^{14}$ e os novos espaços geopolíticos contemporâneos - o que a crítica chilena Nelly Richard vê como uma "multilocalização do sujeito e da crítica" que tem suscitado uma mobilidade de deslocamentos, "capazes de romper os pactos hegemônicos do uniforme e do conforme". ${ }^{15}$ Vale ressaltar que é justamente na complexa interseção entre saber e poder, nos moldes discutidos por Foucault, ou ainda, entre vitimização e agenciamento que podemos entrever muitos dos pontos através dos quais se articulam as relevantes interseções entre os campos teóricos do feminismo e do pós-colonialismo.

Se podemos dizer, por um lado, que o pós-colonialismo se fortalece com a interrupção ocasionada pelos estudos feministas; por outro lado, podemos salientar como a crítica feminista, questionada há algum tempo por seu branqueamento e seu ocidentalismo, tem sido insistentemente levada a refletir, pelas próprias críticas feministas e pelo debate ampliado pelas discussões trazidas pelo pós-colonialismo, sobre a categoria universalista da mulher, abrindo caminho para se teorizarem várias outras e novas formas de se pensarem o lugar das mulheres na contemporaneidade, a falácia da universalidade, a diferença entre as mulheres, os vários sujeitos do feminismo, a transversalidade do gênero, entre outros. No entanto, como observa Ania Loomba, ainda hoje "a palavra 'feminismo' e as agendas popularmente associadas ao feminismo ocidental são vistas com profundo ceticismo no âmbito de vários movimentos de mulheres pós-coloniais". ${ }^{16}$ Perdura, assim, apesar das várias agendas em comum, como vimos, um sentimento recíproco de desconfiança e ceticismo entre teóricos/as feministas e pós-coloniais que faz com que se acirrem, por vezes, debates que seriam mais bem articula- 
17 Para uma discussão sobre os encontros coloniais por um viés que perpassa a crítica feminista, ver PRATT, 1992; e MCCLINTOCK, 1995.

${ }^{18}$ Leela GANDHI, 1998, p. 98.
${ }^{20}$ Heloisa Toller GOMES, 2012.

19 SCHMIDT, 2006, p. 783-784. dos se houvesse, de fato, conhecimento aprofundado sobre suas bases teóricas, bem como disposição e abertura para um diálogo fecundo e uma interação crítica contínua. Para Leela Gandhi, uma área produtiva para se pensar em uma possível colaboração entre o pós-colonialismo e o feminismo seria a ofensiva comum "contra o mito agressivo da masculinidade tanto imperial como nacionalista" por meio da releitura, como vários críticos têm se proposto a fazer, ${ }^{17}$ do encontro colonial como "um conflito entre masculinidades em disputa". ${ }^{18}$

O comentário de Loomba acerca do ceticismo entre as duas tendências teóricas traz à tona outro tipo de descrença que impera no cenário dos estudos pós-coloniais e feministas, especialmente na conjuntura brasileira. No referido artigo "Refutações ao feminismo: (des)compassos da cultura letrada brasileira", no qual avalia em um relato preciso e arguto os entraves enfrentados por uma devida inserção da crítica feminista na cena brasileira, Rita Schmidt observa que

A realidade é que, fora do circuito de suas praticantes, a crítica feminista nem sequer existe, e quando mencionada é considerada com descrédito, muitas vezes com preconceito explícito, e freqüentemente com suspeita, como sendo mais uma teoria de "de fora", expressão que, como já referi, inscreve a crítica feminista no cenário de repúdio ao mimetismo teórico, questão que é associada à tendência brasileira de angariar prestígio intelectual pelo endosso de nomes difíceis e de teorias estrangeiras. ${ }^{19}$

Argumento similar pode ser vislumbrado na avaliação que Heloisa Toller Gomes faz sobre o pós-colonialismo no contexto brasileiro, em "Crítica pós-colonial e o(s) colonialismo(s) europeu(s)":

Análises recentes do colonialismo comparativo (dentro ou fora do "Pós-Colonialismo" institucionalizado) vêm sendo empreendidas em nosso país por um crescente número de pesquisadores. Esta crítica clama pela elaboração de um pensamento pós-colonial que, a exemplo das ciências sociais desde a década de 1930, aprofunde a questão da formação social brasileira e de suas vinculações internacionais através da utilização inteligente da moderna crítica nacional e internacional, sem eurocentrismos fascinados. Isto seria ceder à armadilha de novo colonialismo cultural - desta vez, via academia. ${ }^{20}$

Chama a atenção, sem causar estranheza, a confluência das avaliações sobre a situação brasileira com relação tanto ao feminismo quanto ao pós-colonialismo. Em ambos os argumentos, percebe-se a preocupação, por um lado, no caso do feminismo, com sua falta de inserção no 
21 Com relação ao feminismo, Simone Pereira Schmidt (2008, p. 118) observa apropriadamente que é este o impasse no qual se encontra a crítica feminista em seu trabalho tanto acadêmico quanto político: "de um lado, o provincianismo, e de outro, o colonialismo". Para a autora, uma maneira de lidar com o problema seria por meio de uma teorização e de uma prática de tradução que, na visão de Claudia de Lima Costa (2004, p. 191), seria "o lugar privilegiado para uma negociação das diferenças".

${ }^{22}$ Diana BRYDON, 2012a.

\footnotetext{
${ }^{23}$ A perspectiva de uma teorização situada também tem sido discutida por várias críticas feministas, ver Donna HARAWAY, 1988; SCHMIDT, 2006; e SCHMIDT, 2008.
}

\footnotetext{
${ }^{24}$ Ver a discussão de Spivak sobre o pós-colonialismo e os movimentos globais em "Attention: Postcolonialims" (1997/1998).
}

debate teórico no Brasil e, por outro, com relação ao póscolonialismo, com a necessidade de reflexão sobre um pensamento pós-colonial que se insira na experiência e vivência histórica do país. Em comum, constata-se a percepção de que qualquer teorização, seja sobre o feminismo ou sobre o pós-colonialismo, deva necessariamente levar em consideração a localização e o posicionamento desses campos teóricos em uma ancoragem no contexto brasileiro, a partir de uma reflexão local que possa dialogar com as discussões no âmbito global e internacional, procurando, assim, desfazer as assimetrias históricas que levaram a uma estrutura interligada e excludente de poder e conhecimento, questões essas relevantes que surgem com frequência nos debates tanto sobre o pós-colonialismo quanto sobre 0 feminismo. ${ }^{21}$

Em artigo recente intitulado "New Postcolonialisms: Literary Perspectives from Canada and Brazil", a crítica canadense Diana Brydon tece uma linha argumentativa, referente ao pós-colonialismo, que também poderia se aplicar a essa reflexão que coloca em diálogo a crítica feminista e a pós-colonial. Partindo do pressuposto de que tanto o Brasil quanto o Canadá se mantêm em uma posição marginalizada em relação à teorização sobre os estudos pós-coloniais que tende a circular privilegiadamente nos polos hegemônicos de produção de conhecimento, Brydon postula que a própria crítica pós-colonial beneficiar-se-ia enormemente se houvesse um maior engajamento com as perspectivas canadenses e brasileiras que têm circulado recentemente nesses países. Esses posicionamentos trariam em seu bojo o potencial não somente de mudar a forma de lidar com a crítica pós-colonial, em um contexto transnacional, mas também de influir na própria natureza do trabalho teórico sobre o qual se debruçam os estudos póscoloniais. ${ }^{22}$ Os argumentos de Brydon desvelam sua preocupação com uma suposta tendência universalista dos estudos pós-coloniais e a necessidade de um trabalho teórico que se desenvolva a partir de uma perspectiva situada - formulação também cara à crítica feminista ${ }^{23}$ que abrigue as diferenças e as outras vozes por vezes relegadas às margens dos debates teóricos, uma discussão também já muito conhecida e aventada entre nós.

A postura de uma crítica perspectivada e situada torna-se cada vez mais relevante neste momento histórico em que movimentos de migração e mobilidade globais, que perpassam os vários espaços geopolíticos, podem ser vislumbrados como um significante efeito do momento póscolonial, como observa Spivak. ${ }^{24}$ No entanto, cabe ressaltar que, a despeito de motivações e movimentos contrários, predomina em nossos dias um contínuo processo de 
${ }^{25}$ Walter MIGNOLO, 2008, p. 20.

${ }^{26}$ GANDHI, 2009, p. 38.

27 SCHMIDT, 2006, p. 795.

${ }^{28}$ MIGNOLO, 2010, p. 346.

${ }^{29}$ SPIVAK, 1997/1998, p. 166. deslocamento e recolocação da matriz colonial de poder, nos termos discutidos por Walter Mignolo: "Ahora bien, el nuevo orden de conflictos que aparece en la escena mundial no es por cierto des-colonial. Son conflictos que conducen a la formación de un orden policéntrico y capitalista en la que se mantiene, en disputa, la matriz colonial de poder". ${ }^{25} \mathrm{O}$ que Mignolo articula com sendo um projeto descolonial deve, em sua visão, aspirar a se desprender dessa matriz colonial do poder e, consequentemente, de uma ordem policêntrica que hoje ultrapassa várias fronteiras geopolíticas.

Em sentido semelhante, Gandhi, ao avaliar as críticas dirigidas ao pós-colonialismo, destaca a negativa e perniciosa percepção de tendência ao universalismo (também mencionada por Brydon), baseado em uma crença em um bem comum a ser compartilhado por todos, cultivado em nome de uma suposta democracia que, de fato, se constitui como um conceito ingênuo e equivocado, podendo levar a um perigoso neocolonialismo conservador. Gandhi propõe uma reflexão sobre o conceito de democracia como um "tópico crucial de intervenção pós-colonial" e um tropo significativo por sua amplitude ao abranger múltiplos interesses e preocupações. ${ }^{26}$ Não chega a ser surpreendente, portanto, que seja esse apelo a uma visão democrática, direcionado à crítica feminista, que também mova o texto de Schmidt: "Esta é, no meu entender, a maior contribuição que a crítica feminista pode oferecer: produzir um deslocamento em relação ao modelo de democracia instalada no país". ${ }^{27}$

Se Spivak está correta ao afirmar que a póscolonialidade, como a percebemos hoje, é, de fato, um fracasso da descolonização, como acredito que seja, esse movimento crítico de releitura e desestabilização dos discursos e das produções textuais que nos circundam deve ser uma constante em nossa prática de construção democrática e (des)construção crítica e teórica. A esse posicionamento Walter Mignolo se refere como um necessário exercício de uma "gramática da descolonialidade", isto é, "uma luta pela descolonização epistêmica" que perscrute as consequências desse fazer descolonial no sentido ético, político e teórico. ${ }^{28}$ Daí surge a necessidade de adotarmos uma postura que se coadune com essa preocupação que é tanto uma posição ética quanto uma disposição política efetuada por meio de uma atitude de vigília ininterrupta. Estar vigilante, ${ }^{29}$ no sentido que Spivak dá ao termo, significa não apenas estar atenta às forças da matriz colonial de poder, mas também às possíveis construções ilusórias de democracia e engajamento político e ético (no sentido discutido por Gandhi), e às cumplicidades de nossas posições como intelectuais pós-(e des)coloniais e, sobretudo, como feministas. Significa ainda abrir espaço para um aprendizado contínuo a partir do outro, 
30 SPIVAK, 1994a, p. 205.

${ }^{31}$ SPIVAK, 1994b, p. 57.

${ }^{32}$ Argumento articulado a partir da crítica à teorização de Anibal Quijano sobre a colonialidade do poder e a modernidade. María Lugones (2010, p. 371) argumenta que Quijano parece não perceber sua aceitação do significado hegemônico do gênero. daquele ou daquela cujo discurso silenciado corre o risco de ser apropriado e, assim, ainda mais emudecido. Nas palavras de Spivak, é imprescindível insistir em uma postura crítica que se proponha a "esquecer os privilégios da elite pós-colonial num mundo neocolonial". ${ }^{30}$ Refletir sobre histórias legíveis, alternativas e críticas, mantendo essa postura desestabilizadora e intervencionista, torna-se, então, um papel decisivo tanto da crítica pós-colonial quanto da crítica feminista contemporânea.

Podemos, assim, indagar como poderia uma atitude descolonial e feminista por parte da crítica contemporânea fornecer uma história ou leitura alternativas, a partir do lócus específico de enunciação de um sujeito subalterno e de um espaço da subalternidade e da alteridade, mas que também se articule a um possível agenciamento. No entanto, é importante atentar para o fato de que a subalternidade, como operador crítico, não pode nem resvalar para um discurso vitimizante, nem se tornar uma figura fetichizada e exotizada para um suposto consumo, posto que não há qualquer valor em se congelar o sujeito subalterno nesse espaço excludente e destituído de possibilidade de poder e agenciamento ou entregar nas mãos de outrem (geralmente um intelectual do primeiro mundo, apesar de suas supostas boas intenções) 0 destino a ele reservado. Como bem adverte Spivak, em seu influente artigo "Pode o subalterno falar?", publicado inicialmente em 1988, é preciso estar atenta e consciente do papel que desempenhamos como intelectuais para que não caiamos na armadilha de falar pelo outro ou mantê-lo na subalternidade. Deve-se, ao contrário, realizar um trabalho efetivo e, principalmente, ético contra a subalternidade por meio de espaços de escuta qualificada para com o subalterno que não consegue falar porque não pode ser ouvido, sendo essa "a responsabilidade [que] deve unir o chamado da ética a uma resposta". ${ }^{31}$

Deriva desse questionamento da posição ética que se coloca com relação ao subalterno uma necessária e imbricada reflexão sobre a violência ou cegueira epistemológica (nas palavras de Maria Lugones) ou a violência epistêmica (Spivak) que determina certa cumplicidade entre indivíduos e que frequentemente mantém inalteradas as estruturas da colonialidade do poder - em um sistema que Lugones denomina apropriadamente de "sistema moderno/colonial de gênero" ${ }^{32}$ Ao expandir e complicar a teorização sobre o sistema da colonialidade do poder e a modernidade do pensador peruano Anibal Quijano, Lugones argumenta que a lógica da separação categórica entre raça e gênero distorce o que existe na interseção dessas categorias, ou seja, a violência contra as mulheres de cor: "é apenas quando percebemos o gênero e a raça como sendo imbricados ou 
${ }^{33}$ LUGONES, 2010, p. 373-374.

${ }^{34}$ LUGONES, 2010, p. 377-378.

${ }^{35}$ LUGONES, 2010, p. 383-384.

${ }^{36}$ SPIVAK, 2010b, p. 42-43.

${ }^{37}$ LUGONES, 2010, p. 389. amalgamados é que conseguimos, de fato, ver as mulheres de cor". ${ }^{33}$ Ressalte-se que tal sistema não apenas frequentemente ignora a inerente e necessária transversalidade de gênero, como também, por vezes, reforça seu distanciamento por meio da "construção gendrada do conhecimento na modernidade". ${ }^{34}$

Se, como afirma Lugones (seguindo a trilha de Quijano), o sistema de colonialidade, construído historicamente a partir da colonização europeia, não se refere apenas a um domínio sobre uma suposta classificação racial, mas permeia todo o controle imposto sobre o sexo, a subjetividade, a autoridade e o conhecimento, cabe examinarmos como esse sistema é preservado em contextos nos quais um discurso de agenciamento de um sujeito (que permanece silenciado) parece apagar esse histórico. Essa questão revela não apenas a produção "gendrada" do conhecimento, como também o imbricamento desse sistema de gênero com a colonialidade do poder como uma mútua forma constitutiva, bem como o fato de que, como sabemos, tanto a raça quanto o gênero são "ficções poderosas". ${ }^{35}$ Ambos agem para preservar o que Spivak denomina de "heteronormatividade reprodutiva" por meio do papel imposto às mulheres, como "aquelas que detêm o futuro da nação em seu ventre", ao assumirem por um desvio constitutivo a função de dar suporte a uma retórica nacionalista por meio de um investimento ou de uma manipulação do lugar ocupado por essas mulheres. ${ }^{36}$

As questões aqui formuladas, não pretendendo ser conclusivas diante de um tema tão complexo e intricado, se propuseram a fornecer subsídios para a continuidade de uma reflexão necessária e urgente sobre as intersecções entre o(s) feminismo(s) e o(s) pós-colonialismo(s), concebidos necessariamente como plurais. Dessa forma, refletir sobre a responsabilidade ética e um posicionamento crítico no contexto do sistema moderno/colonial de gênero/raça, sobre o qual nos fala Lugones, permitir-nos-ia estabelecer uma articulação pertinente com os lugares de fala das mulheres subalternas e/ou pós-coloniais. É importante também pensarmos nas formas de como se dá a escuta da fala dessas subalternas, com qual sensibilidade, solidariedade e responsabilidade ética, ou mesmo com qual possibilidade de aprendizado ou de trabalho contra subalternidade como uma forma de descolonização do saber. É imprescindível ainda refletir, seguindo mais uma vez as palavras de Lugones, como essa postura torna "visível nossa colaboração com uma sistemática violência de gênero racializada". ${ }^{37}$ Ou seja, como praticar permanentemente uma escuta que não se limite ao exercício de cumplicidade dos intelectuais com os sistemas hegemônicos, como adverte Spivak? Essa postura questionadora nos alerta para nosso posicionamento como intelectuais 
e produtores de saber e conhecimento, e nossa inerente cumplicidade com os discursos que se erigem como críticos, mas que efetuam o silenciamento desse outro por quem pretendemos falar, como procurei discutir. No entanto, é justamente a contrapelo que uma crítica intervencionista e democrática, quer seja ancorada no feminismo ou no póscolonialismo (ou em ambos), e que se instala com base na responsabilidade ética e na solidariedade deve se construir: por meio de uma abertura para as histórias alternativas, legíveis e críticas, conforme preconizam Spivak e Schmidt nas citações que abrem este texto. E, nesse processo, tanto o feminismo quanto o pós-colonialismo, concebidos como teorizações que desestabilizam as bases consolidadas de construção de saber e conhecimento, podem ter um papel decisivo de reflexão teórica e prática efetiva por meio de uma crítica intrinsecamente articulada.

\section{Referências}

ADELMAN, Miriam. "Feminismo e pós-colonialidade: algumas reflexões a partir da teoria social e da literatura". In: WOLFF, Cristina Scheibe et al. Leituras em rede: gênero e preconceito. Florianópolis: Editora Mulheres, 2007. p. 391-413.

ALVAREZ, Sonia E. "Construindo uma política feminista translocal da tradução". Revista Estudos Feministas, v. 17, n. 3, p. 743-753, 2009.

BRYDON, Diana. New Postcolonialisms: Literary Perspectives from Canada and Brazil. Disponível em: <http:// myuminfo.umanitoba.ca>. Acesso em: 30 ago. 2012a. . Post-colonialim Now: Autonomy, Cosmopolitanism, and Diaspora. Disponível em: <http://myuminfo.umanito ba.ca> . Acesso em: 30 ago. 2012b.

COSTA, Claudia de Lima. "Feminismo, tradução, transnacionalismo". In: COSTA, Claudia de Lima; SCHMIDT, Simone Pereira (Org.). Poéticas e políticas feministas. Florianópolis: Editora Mulheres, 2004. p. 187-196.

EAGLETON, Mary. Figuring the Woman Author in Contemporary Fiction. London: Palgrave, 2005.

FRIEDMAN, Susan Stanford. Mappings: Feminism and the Cultural Geographies of Encounter. Princeton: Princeton University Press, 1998.

GANDHI, Leela. Postcolonial Theory: A Critical Introduction. New York: Columbia University Press, 1998.

. 'The Pauper's Gift': Postcolonial Theory and the New Democratic Dispensation." Public Culture, v. 23, n. 1, p. 27-38, 2009.

GOMES, Heloisa Toller. A crítica pós-colonial e o(s) colonialismo(s) europeus. Disponível em: <www.pacc.ufrj.br/z/ano3/01/ artigo04.htm >. Acesso em: 30 ago. 2012. 
GREWAL, Inderpal; KAPLAN, Caren (Ed.). Scattered Hegemonies: Postmodernity and Transnational Feminist Practices. Minneapolis: University of Minnesota Press, 1994.

HALL, Stuart. "Cultural Studies and its Theoretical Legacies." In: MORLEY, David; CHEN, Kuan-Hsing (Ed.). Stuart Hall: Critical Dialogues in Cultural Studies. London: Routledge, 1996. p. 262-275.

HARAWAY, Donna. "Situated Knowledges: The Science Question in Feminism and the Privilege of Partial Perspective." Feminist Studies, v. 13, n. 3, p. 575-599, 1988.

HOLLANDA, Heloisa Buarque de. Pós-feminismo. 1994. Disponível em: <www.heloisabuarquedehollanda.com. br>. Acesso em: 30 out. 2010.

HOOKS, bell. Yearning: Race, Gender, and Cultural Politics. Boston: South End Press, 1990.

LOOMBA, Ania. Colonialism/Postcolonialims. London/Nova lorque: Routledge, 1998.

LUGONES, María. "The Coloniality of Gender." In: MIGNOLO, Walter D.; ESCOBAR, Arturo. Globalization and the Decolonial Option. London/New York: Routledge, 2010. p. 369-399.

MACHADO, Lia Zanotta. "Gênero, um novo paradigma?". Cadernos Pagu, v. 11, p. 107-125, 1998.

McCLINTOCK, Anne. Imperial Leather: Race, Gender and Sexuality in the Colonial Contest. London: Routledge, 1995.

MIGNOLO, Walter D. "La opción descolonial". Letral, n. 1, p. 4-22, 2008.

. "Delinking: The Rhetoric of Modernity, the Logic of Coloniality and the Grammar of De-coloniality." In: MIGNOLO, Walter D.; ESCOBAR, Arturo. Globalization and the Decolonial Option. London/New York: Routledge, 2010. p. 303-368.

MORAGA, Cherríe; ANZALDÚA, Gloria (Ed.). This Bridge Called My Back: Writings by Radical Women of Color. New York: Kitchen Table Press, 1981.

PRATT, Mary Louise. Imperial Eyes: Travel Writing and Transculturation. London: Routledge, 1992.

RICHARD, Nelly. Intervenções críticas: arte, cultura, gênero e política. Belo Horizonte: UFMG, 2002.

SANTIAGO, Silviano. O cosmopolitismo do pobre: crítica literária e crítica cultural. Belo Horizonte: UFMG, 2004.

SCHMIDT, Rita Terezinha. "Refutações ao feminismo: (des)compassos da cultural letrada brasileira". Revista Estudos Feministas, Florianópolis, v. 14, n. 3, p. 765-799, 2006.

. "Revisitando a mulher na literatura: horizontes e desafiOs". In: STEVENS, Cristina. Mulher e literatura - 25 anos: raízes e rumos. Florianópolis: Editora Mulheres, 2010. p. 257-270. 
SCHMIDT, Simone Pereira. "A seção debates em revista: práticas feministas de tradução". Revista Estudos Feministas, v. 16, n. 1, p. 117-122, 2008.

SPIVAK, Gayatri Chakravorty. "Who Claims Alterity?" In: KRUGER, Barbara; MARIANI, Phil (Ed.). Remaking History: Discussion in Contemporary Culture. Seattle: Bay Press, 1989. p. 269-92.

. "Quem reivindica alteridade?". Tradução de Patricia Silveira de Farias. In: HOLLANDA, Heloisa Buarque de. Feminismo em tempos pós-modernos. Rio de Janeiro: Rocco, 1994a. p. 187-205.

. "Responsibility." Boundary 2: An International Journal of Literature and Culture, Durham, NC, v. 21 , n. 3, p. 1964, 1994b.

. "Attention: Postcolonialism!" Journal of Caribbean Studies, v. 12, n. 2/3, p. 159-170, 1997/1998.

. A Critique of Postcolonial Reason: Toward a History of the Vanishing Present. Cambridge, Massachusetts: Harvard University Press, 1999.

. Pode o subalterno falar? Tradução de Sandra Regina Goulart Almeida, Marcos Pereira Feitosa e André Pereira Feitosa. Belo Horizonte: UFMG, 2010a.

. Nationalism and the Imagination. London/New York/ Calcutta: Seagull Books, $2010 \mathrm{~b}$.

[Recebido e aceito para publicação em abril de 2013]

\section{Feminist Interventions: Postcolonialim, Power and Subalterneity}

Abstract: The article proposes to promote a discussion on postcolonial theory and feminist criticism with an aim to discussing its articulation and specificities, and also to thinking its relation to the critical theories produced in Brazil.

Key Words: Postcolonialism; Feminism; Subalterneity. 